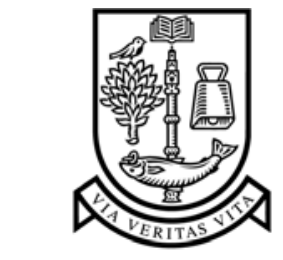 \\ UNIVERSITY \\ of \\ GLASGOW
}

Brown, L.M. and Brewster, S.A. and Purchase, H.C. (2006)

Multidimensional tactons for non-visual information presentation in mobile devices. In, 8th conference on Human-Computer Interaction with Mobile Devices and Services, 12-15 September 2006 ACM International Conference Proceeding Series Vol 159, pages pp. 231-238, Helsinki, Finland.

http://eprints.gla.ac.uk/3256/ 


\title{
Multidimensional Tactons for Non-Visual Information Presentation in Mobile Devices
}

\author{
Lorna M. Brown, Stephen A. Brewster and Helen C. Purchase \\ Glasgow Interactive Systems Group \\ Department of Computing Science, University of Glasgow, G12 8QQ, UK \\ \{lorna, stephen, hcp\} @dcs.gla.ac.uk
}

Phone: +44 1413304256

\begin{abstract}
Tactons are structured vibrotactile messages which can be used for non-visual information presentation when visual displays are limited, unavailable or inappropriate, such as in mobile phones and other mobile devices. Little is yet known about how to design them effectively. Previous studies have investigated the perception of Tactons which encode two dimensions of information using two different vibrotactile parameters (rhythm and roughness) and found recognition rates of around $70 \%$. When more dimensions of information are required it may be necessary to extend the parameter-space of these Tactons. Therefore this study investigates recognition rates for Tactons which encode a third dimension of information using spatial location. The results show that identification rate for three-parameter Tactons is just $48 \%$, but that this can be increased to $81 \%$ by reducing the number of values of one of the parameters. These results will aid designers to select suitable Tactons for use when designing mobile displays.
\end{abstract}

\section{Categories and Subject Descriptors}

H5.2. User Interfaces: Haptic I/O.

\section{General Terms}

Design, Human Factors.

\section{Keywords}

Tactile icons, Tactons, Tactile displays, non-visual interaction.

\section{INTRODUCTION}

When interacting with mobile devices, such as mobile phones and personal digital assistants (PDAs), the visual display is often insufficient due to the limited screen space, or inappropriate, such as when the device is in a pocket but requires the user's attention. Therefore it is important to consider using alternative modalities through which information can be presented.

This research investigates the possibilities of using the tactile

Permission to make digital or hard copies of all or part of this work for personal or classroom use is granted without fee provided that copies are not made or distributed for profit or commercial advantage and that copies bear this notice and the full citation on the first page. To copy otherwise, or republish, to post on servers or to redistribute to lists, requires prior specific permission and/or a fee.

Conference'04, Month 1-2, 2004, City, State, Country.

Copyright 2004 ACM 1-58113-000-0/00/0004 ...\$5.00. modality to present information in alerts in mobile devices. Vibrotactile displays have been featured in mobile devices such as mobile phones and PDAs for many years, and are used to alert users to incoming calls or upcoming calendar appointments. However, these alerts have generally only consisted of a simple buzz to indicate that the user's attention is required and do not provide any information about what the user is being alerted to. The cutaneous sense is capable of processing much more complex tactile patterns, and by utilizing this capability it would be possible to present more detailed information in these alerts.

In recent years manufacturers have begun to use more complex vibrotactile patterns in their products, for example Immersion has developed VibeTonz technology [1] for enhancing ringtones and games in mobile phones, while Motorola use a multi-function transducer to enhance ringtones with haptic effects [6]. While these products do make use of more complex vibrations, they generally only use the vibration to enhance the audio alerts.

Using tactile feedback alone, without audio feedback, would make it possible to communicate information discretely without disturbing other people or to alert users while they are in noisy environments. For example, tactile alerts could be used to provide users with detailed information about incoming calls (e.g caller, importance of call, type of call/message) or about upcoming appointments in a calendar application (e.g. type of appointment, location, importance and time). By providing such alerts via vibrotactile messages it would be possible for users to discretely monitor incoming calls and upcoming calendar alerts without having to look at the device, meaning that they could choose to only respond to those alerts that they are interested in.

This research investigates the design of vibrotactile messages, called Tactons [2], which can be used to communicate complex information through the tactile modality alone.

\subsection{Tactons}

Tactons [2] are structured, tactile messages which can be used to communicate information non-visually. Information is encoded in Tactons by manipulating parameters of vibration. Several dimensions of information can be represented in a single Tacton by encoding each dimension in a different vibrotactile parameter.

Previous research identified rhythm and vibrotactile "roughness" as suitable parameters for Tactons, and showed that Tactons encoding two dimensions of information in these parameters can be understood by users, with recognition rates of around 70\% [3]. To expand the design space to allow a wider range of Tactons to be created, it is necessary to identify a third vibrotactile parameter in which another dimension of information can be encoded. 
Therefore, this research aims to identify a suitable third parameter, and to investigate recognition rates achieved for the resulting 3-Parameter Tactons when an absolute identification experiment is carried out.

\section{SELECTING VIBROTACTILE PARAMETERS}

This section describes the vibrotactile parameters which have been shown to be successful in previous studies and discusses the choice of a third parameter for encoding another dimension of information in Tactons.

\subsection{Vibrotactile Actuator}

The parameters of vibration which can be used are dependent on the actuators used to produce the vibrations. The actuators used in this research were C-2 Tactors from Engineering Acoustics Incorporated (www.eaiinfo.com). The C-2 Tactor (Figure 1) is a moving magnet, linear vibration motor, designed to be resonant at $250 \mathrm{~Hz}$. The contactor is mounted above the housing and preloaded against the skin. These devices were used in previous studies on Tactons encoding two dimensions of information [3].

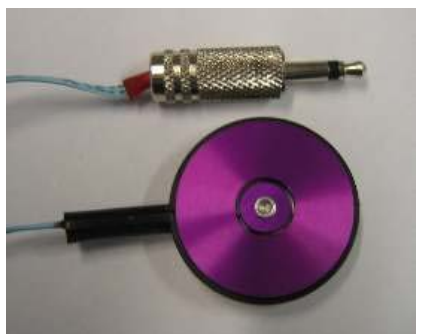

Figure 1: The C2 Tactor from Engineering Acoustics Inc (www.eaiinfo.com).

\subsection{Existing Parameters}

Previous studies have identified rhythm and roughness as suitable vibrotactile parameters. This section explains how these parameters are created and how they could be used.

\subsubsection{Rhythm}

Rhythm has been identified as a successful parameter in Tacton design, with recognition rates of over $90 \%$ achieved when three different rhythms are used [3]. Rhythms are created by grouping together vibrotactile pulses of different durations, and leaving gaps between them to create patterns in the same way that a rhythm might be created in music by combining notes and rests.

\subsubsection{Roughness}

A previous study showed that the sensation of vibrotactile "roughnesses" can be created using amplitude modulated waveforms, and that roughness can be used successfully as a parameter in Tactons, with recognition rates of around $80 \%$ when three levels are used [3]. Amplitude modulated signals are created by multiplying a sine wave of a given frequency by a sine wave of another frequency, thus modulating the amplitude of the base signal by the second frequency. Figure 2 shows a $250 \mathrm{~Hz}$ sinusoid modulated by a $30 \mathrm{~Hz}$ sinusoid to create a "very rough" sensation.

\subsection{Selecting a Third Parameter}

To select a third parameter for encoding information in Tactons it is necessary to consider the remaining vibrotactile parameters that are available. A suitable parameter would be one in which users can identify multiple levels, and that will not degrade perception of the existing parameters when combined with them. This section considers the possible parameters that could be selected.

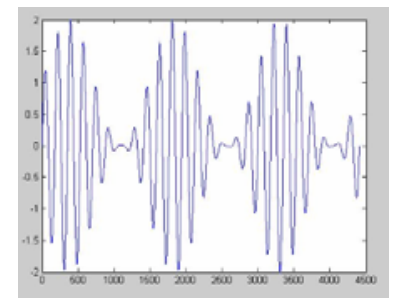

Figure 2: A $250 \mathrm{~Hz}$ sine wave modulated by $30 \mathrm{~Hz}$ to create a "very rough" sensation.

\subsubsection{Frequency}

While humans can hear sounds in the range $20-20,000 \mathrm{~Hz}$, the practical frequency range of the skin is much smaller, ranging from $10 \mathrm{~Hz}$ to $400 \mathrm{~Hz}$ [7]. The usable frequency range is further reduced by the limited bandwidth of actuators such as the C-2, which are designed to be resonant at $250 \mathrm{~Hz}$ rather than to produce a wide range of frequencies. Therefore, frequency is unsuitable as a parameter in Tacton design when using most common vibrotactile actuators.

\subsubsection{Intensity}

A previous study found that intensity could be used successfully as a parameter in Tactons when three levels of intensity were presented using a standard mobile phone vibration motor [5]. In addition it has been shown that intensity changes over time can be identified by users [4]. While both of these intensity manipulations might be used successfully in Tactons when using the C-2 Tactors, using intensity in combination with roughness could be problematic since both parameters are created by manipulating the amplitude of the vibration. Since the aim of this study was to find a third parameter which could be used in combination with the existing parameters (rhythm and roughness), intensity would not be a suitable choice (future studies might investigate using intensity in place of roughness, or alongside it, to provide redundancy).

\subsubsection{Spatial Location}

The spatial location of transducers on the body has been used successfully by many researchers $[13,14]$. Presenting Tactons to different spatial locations may degrade perception of the other parameters due to the differing tactile sensitivity at different body sites. However, it seems likely that any degradation caused by this would be less than that caused by altering another parameter of the single point stimulus (such as intensity, as described above).

In order to use spatial location as a parameter in Tactons, it is important to choose the locations carefully. Cholewiak and Collins [8] report that early work on tactile perception suggested that tactile localization is most precise when the stimulus is close to an anatomical reference point, and in particular at points of mobility such as the wrist or elbow. When investigating vibrotactile localization performance with an array of seven 
Tactors on the forearm, Cholewiak and Collins [8] found that perception at the wrist and elbow was more precise than that for the rest of the array, indicating that these early findings transfer to vibrotactile stimuli. Cholewiak et al. [9] also conducted a study on the abdomen, where the main anatomical references are the spine and navel, and found that again localization was most precise when the stimuli occurred at these reference points. In addition they found that people were unlikely to mistake stimulation at another point for stimulation at one of the reference points.

These findings suggest that for accurate localization of three locations, two Tactors should be located at anatomical reference points, with the remaining Tactor located at a point between these two. Based on the above findings, localization should be accurate at the reference points, and stimulation at the non-reference point is unlikely to be confused with stimulation at reference points.

In addition to using static locations as described above, the use of multiple Tactors would enable the use of spatio-temporal patterns. By activating different Tactors in turn, the sensation of movement in different directions across the skin could be provided [13].

\subsection{Discussion}

Based on the information regarding the available parameters discussed above, static spatial location was selected as a suitable parameter to be combined with the existing two parameters, roughness and rhythm. As the arm is a practical location on which to attach Tactors, the arm was selected for the studies reported in these papers, but future studies could investigate the presentation of Tactons to points around the abdomen. Section 3 discusses how these parameters were combined to create 3-Parameter Tactons.

\section{DESIGN OF 3-PARAMETER TACTONS}

In this study Tactons were created to represent alerts which might occur in an electronic diary to remind the user of an upcoming appointment. Three pieces of information were encoded in each Tacton using the parameters identified in Section 2: the type of appointment was encoded in the rhythm, the importance of the appointment was encoded in the roughness, and time remaining until the appointment was encoded in the location of the vibration on the user's forearm. Each piece of information had three possible values: the type of appointment could be a Meeting, a Lecture or a Tutorial, the importance could be Low, Medium or High and the time until the appointment could be 30 minutes, 15 minutes or 5 minutes. This resulted in a set of 27 Tactons.

\subsection{Type of Appointment (Rhythm)}

Three different rhythms, which had been shown to be successful in a previous study [3], were used to represent the three types of appointment: meeting, lecture and tutorial. Each rhythm was made up of a different number of pulses, with the meeting rhythm made up of seven short pulses, the lecture rhythm of four longer pulses, and the tutorial rhythm of one short pulse and one very long pulse. Using a different number of pulses (or beats) in each rhythm should help to make the rhythms distinguishable. These rhythms are presented in Figure 3, Figure 4 and Figure 5 using standard musical notation for rhythm, on a single line since no pitch information is required.



Figure 3: Meeting Rhythm.

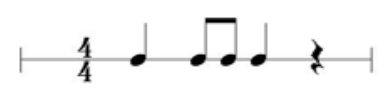

Figure 4: Lecture Rhythm.

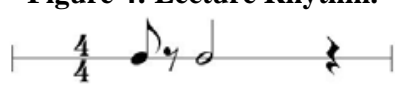

Figure 5: Tutorial Rhythm.

\subsection{Importance of Appointment (Roughness)}

Three distinct roughnesses were used to represent low (smooth), medium (rough) and high (very rough) importance appointments. The three amplitude modulations chosen, based on the previous study, were an un-modulated $250 \mathrm{~Hz}$ sine wave (smooth), and the same sine wave modulated by $50 \mathrm{~Hz}$ (rough) and $30 \mathrm{~Hz}$ (very rough, Figure 2).

\subsection{Time Until Appointment (Spatial Location)}

Three locations on the user's volar forearm were used to encode information about the time remaining before the appointment the wrist, the elbow, and the point equidistant between these two reference points. Based on Cholewiak's findings reported in Section 2.3.3 the reference points (wrist and elbow) should be accurately localized, and the third point should not be confused with either of the two reference points, therefore near-perfect performance should be possible.

C-2 Tactors were attached to the user's forearm at these three points using Velcro bands. The "wrist" Tactor was positioned $1 \mathrm{~cm}$ from the participant's wrist, the "elbow" Tactor $1 \mathrm{~cm}$ from the participant's elbow and the "mid-point" Tactor at a point equidistant between these two Tactors (Figure 6).

A vibration at the wrist indicated that the appointment was due to occur in 5 minutes, a vibration at the mid-point that it would occur in 15 minutes, and a vibration at the elbow that the appointment would take place in 30 minutes.



Figure 6: The location of the Tactors on the participant's forearm.

\subsection{Examples}

Three examples will explain this mapping in more detail: 1: A High Importance Meeting in 5 minutes would be represented by the Meeting Rhythm and a "very rough" vibration and would be presented to the wrist.

2: A Low Importance Meeting in 5 minutes would be represented by the Meeting Rhythm and presented to the wrist (as above), but would be represented by a "smooth" vibration.

3: A Low Importance Lecture in 30 minutes would be represented by the Lecture Rhythm and a "smooth" vibration, and would be presented to the elbow. 


\section{EVALUATION OF 3-PARAMETER TACTONS}

An experiment was conducted to investigate absolute identification of Tactons encoding three dimensions of information. The Tactons described in Section 3 were presented to users and data were recorded on the identification of complete Tactons (correct identification of all three parameters - type, importance and time until appointment), in addition to the recognition of the individual attributes.

\subsection{Hypotheses}

The aim of this experiment was to investigate the recognition rates for 3-parameter Tactons in an absolute identification experiment. A previous study evaluating Tactons encoding two parameters of information found a recognition rate of $71 \%$ for complete Tactons [3]. For the individual parameters, a recognition rate of $93 \%$ was found for rhythm, while roughnesses were correctly identified $80 \%$ of the time. Differences in the hardware set-up mean that a direct comparison to the previous study is not possible but some general comparisons can be made between the results.

Adding a third parameter to Tactons is likely to degrade perception of the existing parameters, therefore it was expected that the overall identification performance on Tactons would decrease. In particular, the fingertip (which was used in the previous study) is one of the most sensitive parts of the body for perceiving tactile stimuli [10] and is therefore likely to be the most sensitive to subtle variations in vibration such as roughness. The forearm contains less receptors and is therefore less likely to be as sensitive to these subtleties. Rhythm, on the other hand, is less likely to be affected by the change in location as it does not require subtle discriminations to be made, therefore comparable performance to that achieved in the previous study (i.e. over 90\%) was expected. Performance for the spatial location parameter was expected to be close to perfect (over 90\%), due to the careful choice of locations as discussed previously.

Based on this information, the hypotheses were as follows:

1. The identification of complete Tactons will be lower for threeparameter Tactons than for two-parameter Tactons.

2. Rhythms will be correctly identified over $90 \%$ of the time.

3. Perception of roughness will be lower for three-parameter Tactons than for two-parameter Tactons.

4. Locations will be correctly identified over $90 \%$ of the time.

\subsection{Hardware set-up}

Three C-2 Tactors were attached to the participant's nondominant forearm using Velcro bands (Figure 6) at the positions described in Section 3.3. The participant's dominant arm was left free to control the mouse, which was used to make responses. The participant wore closed-back headphones to block out any noise from the Tactors.

Tactile sensitivity varies across the length of the forearm, therefore the intensity of the vibrations could feel very different at different points on the arm, even if the actual output levels were carefully controlled [8]. These differences in intensity could be used by participants to aid in localizing the vibrations, rather than just using the spatial location. Therefore, before starting the experiment it was necessary for each participant to set the levels of the three Tactors so that they all felt perceptually equivalent. The intensity of the mid-point transducer was fixed, but participants were able to alter the strength of the vibrations at the elbow and wrist Tactors. They could alter the strength by turning the volume control on an amplifier up and down for each Tactor until they felt that the strength perceptually matched the strength of the vibration at the mid-point Tactor.

\subsection{Experimental Design}

16 participants took part in this experiment and were paid $£ 6$. The participants were all students or staff of the University of Glasgow and were aged 17 to 36 (mean=23). Six of the participants were male, while 10 were female. Two were left handed and the rest were right handed.



Figure 7: The Tacton training interface.

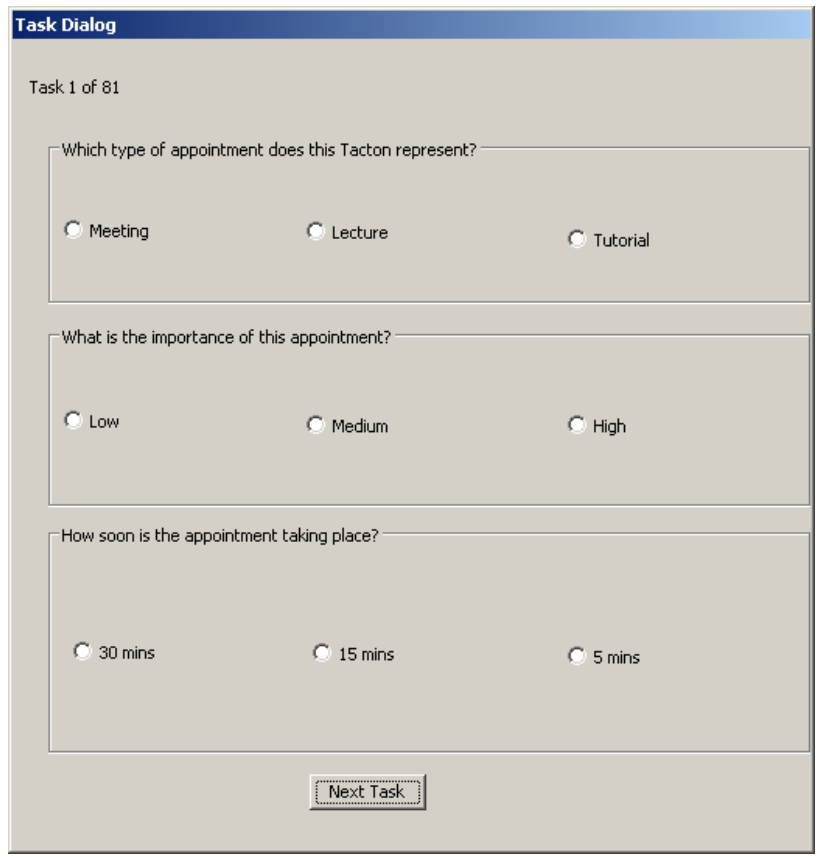

Figure 8: The response interface.

Before beginning the experiment participants were introduced to the concept of Tactons, and the mapping between the vibrotactile parameters and the attributes of the appointment alerts were 
explained. They were then allowed to try out the Tactons by themselves for up to 10 minutes, by clicking on buttons on a dialogue box (Figure 7). They were advised to use this time to learn the mapping between the Tactons and their meanings before being tested on these in the experiment. After familiarising themselves with the Tactons, they took part in 27 tasks like those from the experiment itself as training in how to use the interface.

There were 81 tasks in the experiment itself, with all 27 Tactons presented three times within the experiment. The order in which the Tactons were presented was randomized for each participant. In each task the participant was presented with one Tacton, which was repeated four times with a one second pause between repetitions. While the Tacton was being presented, the participants had to identify all three attributes encoded in the Tacton, by clicking the corresponding radio buttons on a dialogue box (Figure 8). They did not need to wait until the Tacton had been presented all four times before responding if they had made a decision before then. When they had made their decision they could click the "Next Task" button to enter their response.

\subsection{Results}

During the experiment data were collected on the number of correct responses to the attributes of each Tacton, and percentage correct scores were calculated for each individual attribute and for the complete Tactons. In addition the information transmitted for the complete Tactons and for the individual parameters was calculated from stimulus-response confusion matrices [12]. Three participants were removed from the analysis due to outlier behavior (their percentage correct scores for one or more attributes were more than two standard deviations away from the mean) so the results reported here are for the remaining 13 .

\subsubsection{Percentage correct scores}

The results for overall Tacton recognition showed an average recognition rate of $47.8 \%$ (standard deviation $=15.1$ ). The best performance was achieved for the High Importance Lecture in 15 Minutes, which had a recognition rate of $74.4 \%$, while the Low Importance Tutorial in 5 Minutes had the lowest recognition at just $15.4 \%$.

Appointment types (represented by rhythm) were correctly recognised on average $96.7 \%$ of the time. The identification rates for each rhythm are shown in Table 1 . The results for recognition of importance of appointment (represented by roughness) showed an average recognition rate of $50.2 \%$. Table 2 shows the individual recognition rates for each level of roughness. The results for recognition of time until appointment (represented by location) showed an average recognition rate of $95.5 \%$, with recognition of all three values above $95 \%$, as shown in Table 3.

The percentage correct score for complete Tactons (47.8\%) is very low compared to the results for two-parameter Tactons (71\%), from [3]. In addition, the percentage correct score for roughness $(50.2 \%)$ is also much lower than the $80 \%$ achieved for two-parameter Tactons. Differences in the hardware setup mean that a direct comparison is not completely fair, and therefore no statistical analysis has been performed on these data, however these two results indicate that it might be possible to accept Hypotheses 1 and 3. The percentage correct scores for both rhythm (96.7\%) and spatial location are both over 90\% and therefore Hypotheses 2 and 4 can be accepted.
These results suggest that roughness is the main parameter which degrades the performance on identification of complete Tactons. By examining the information transmission results it is possible to identify how many values of each parameter should be used to achieve near-perfect performance.

\begin{tabular}{|l|l|l|l|}
\hline & Meeting & Lecture & Tutorial \\
\hline \%correct & 94.87 & 97.15 & 98.01 \\
\hline SD & 14.15 & 5.05 & 3.58 \\
\hline
\end{tabular}

Table 1: Percentage correct scores for Type parameter (rhythm), with standard deviations (SD)

\begin{tabular}{|l|l|l|l|}
\hline & $\begin{array}{l}\text { Low } \\
\text { (Smooth) }\end{array}$ & $\begin{array}{l}\text { Med } \\
\text { (Rough) }\end{array}$ & $\begin{array}{l}\text { High } \\
\text { (Very Rough) }\end{array}$ \\
\hline \%correct & 36.18 & 49.57 & 64.96 \\
\hline SD & 16.30 & 9.75 & 21.84 \\
\hline
\end{tabular}

Table 2: Percentage correct scores for importance parameter (roughness), with standard deviations (SD)

\begin{tabular}{|l|l|l|l|}
\hline & $\begin{array}{l}\text { 30mins } \\
\text { (elbow) }\end{array}$ & $\begin{array}{l}\text { 15mins } \\
\text { (mid-point) }\end{array}$ & $\begin{array}{l}\text { 5mins } \\
\text { (wrist) }\end{array}$ \\
\hline \%correct & 95.7265 & 95.1567 & $\begin{array}{l}95.726 \\
5\end{array}$ \\
\hline SD & 14.33 & 12.23 & 14.33 \\
\hline
\end{tabular}

Table 3: Percentage correct scores for time-until-appointment parameter (location), with standard deviations (SD)

\subsubsection{Information transmission}

Information transmission (also known as "uncertainty reduction" [12]) is a measure of the correlation between the amount of information in the stimuli and the amount of information in the responses given by the participant [11]. Miller [11] suggests that this can be considered as two overlapping circles (Figure 9), with the left circle showing the information input (the amount of information in the stimuli), the right circle showing the information output (the amount of information in the participant's responses), and the overlapping section the information transmitted (the information in the user's response which matches that in the stimuli).

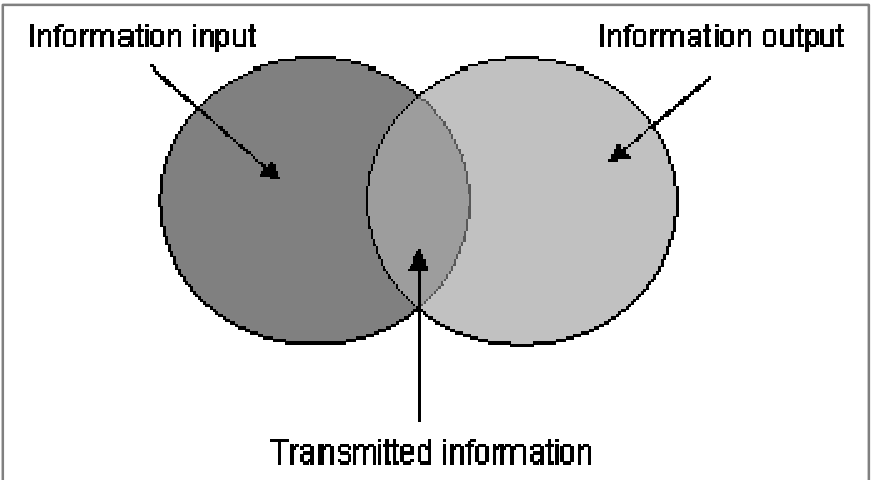

Figure 9: Information Transmission Diagram adapted from a description by Miller [11] 
Information transmitted is measured in bits. The number of bits is calculated by taking the logarithm (to base 2) of the number of alternatives available for the user to choose from. For example there are 27 Tactons in the set used in this experiment and therefore the maximum information available from this set of Tactons is 4.75 bits. Once the information transmitted has been calculated it is also possible to calculate the number of stimuli which should be used to achieve near-perfect performance. This is calculated by raising two to the power of the number of bits (e.g. if the information transmitted via the rhythm parameter was 1 bit, the number of rhythms that should be used if near perfect performance was to be achieved would be 2). In this paper every time an amount of information is reported in bits, the corresponding number of stimuli that this refers to is also reported. These are referred to as "tokens".

As stated above, the maximum information available from the set of Tactons was 4.75 bits (27 tokens) while, for each individual parameter, a maximum of 1.58 bits ( 3 tokens) was available. The actual information transmitted in this experiment was calculated for the complete set of Tactons and for the individual parameters using the stimulus-response confusion matrices. The results showed that the information transmitted for the complete set of Tactons was 2.98 bits (7.9 tokens). For the individual parameters, the information transmitted through the type parameter (rhythm) was 1.36 bits (2.6 tokens), through the importance parameter (roughness) was 0.12 bits (1.09 tokens), and through the time until appointment parameter (location) was 1.28 bits (2.43 tokens). The stimulus-response confusion matrix for roughness is shown in Table 4.

\begin{tabular}{|l|r|r|r|}
\hline & \multicolumn{1}{l|}{ low } & \multicolumn{1}{l|}{ med } & \multicolumn{1}{l|}{ high } \\
\hline low & 127 & 143 & 81 \\
\hline med & 88 & 174 & 89 \\
\hline high & 35 & 88 & 228 \\
\hline
\end{tabular}

Table 4: The Stimulus-Response Confusion Matrix for the importance parameter (represented by roughness)

The information transmitted through complete Tactons indicates that perfect performance could have been achieved if only 7 or 8 Tactons had been used rather than the 27 in this experiment, as 7.9 tokens $(2.98$ bits $)$ were transmitted. The information transmission results for rhythm and location suggest that nearperfect performance could have been achieved for both of these parameters if only two levels of each had been used. The number of bits of information transmitted through the roughness parameter was 0.12 bits. Since the corresponding number of tokens is less than two this indicates that it may not be possible to achieve near-perfect performance with two or more roughnesses. However, reducing the number of roughnesses to two should help to improve performance and this is explored in the following sections.

\subsection{Discussion}

The overall identification rate of $47.8 \%$, and the information transmission of just 2.98 bits ( 7.9 tokens) out of a possible 4.75 bits (27 tokens) is very low, therefore ways in which performance can be improved must be considered.

Reducing the amount of information encoded in the Tactons should reduce confusion, and therefore increase the identification rate and increase the amount of information transmitted. The identification rates of over $95 \%$ for both rhythm and location are very high therefore the three values of these parameters can be retained. The performance on roughness, however, indicates that three levels cannot be distinguished. Although the information transmission results indicate that it might not be possible to achieve perfect performance using two levels of roughness, reducing the number of roughnesses to two is a reasonable step towards improving performance whilst retaining a parameter with some use. Therefore, the next experiment aimed to investigate the effect of reducing the number of roughnesses to two.

\section{IMPROVING THE DESIGN OF 3- PARAMETER TACTONS}

Based on the results of the experiment reported in Section 4, the number of roughnesses used in the 3-parameter Tactons was reduced from three to two, and a second experiment was carried out to investigate whether performance improved as a result.

The smooth (sine) and very rough (sine wave modulated by $30 \mathrm{~Hz}$ ) roughnesses were used in this experiment, to represent low and high importance appointments. These two roughnesses were selected as they were at two ends of the scale - one smooth and one very rough - and should therefore be the two most perceptually different stimuli. Reducing the number of roughnesses to two resulted in a set of 18 possible Tactons.

\subsection{Aim and Hypotheses}

This experiment investigated whether performance improves when the number of roughnesses is reduced from three to two. In addition to improving the percentage correct score, it is possible that reducing the number of roughnesses could reduce confusion and therefore increase the amount of information transmitted, even though less information is input.

The hypotheses were that reducing the number of roughnesses from three to two would result in:

1. a significant improvement in the percentage correct score for complete Tactons.

2. a significant improvement in the percentage correct score for the roughness parameter.

3. an increase in the amount of information transmitted.

4. comparable performance on identification of rhythm and location to that found in the previous experiment.

\subsection{Experimental Design}

Six participants who had taken part in the three-parameter experiment were selected at random and asked to return to take part in this follow-on study. This took place within the two weeks following the original experiment. They were again paid $£ 6$ for their participation. Five of the six participants were male, and one was female, and they were aged between 19 and 32 (mean=24). All were right handed. The results in this follow-on experiment can be compared back to the results of the same six participants in the original study for a within-groups comparison

The experimental procedure was replicated exactly from the previous experiment. Participants were again given ten minutes to familiarise themselves with the set of Tactons, but most participants chose to start the experiment after around two 
minutes as they were already familiar with the Tactons from the previous experiment. Since there were only 18 Tactons in this set, there were 18 tasks in the training and 54 in the experiment itself (three repetitions of each Tacton). As in the previous experiment the order of presentation of the Tactons was randomised for each participant.

\subsection{Results}

Before reporting the results of this experiment it is important to examine the results of these six participants in the original experiment. In the previous experiment these six participants achieved a recognition rate of $48.8 \%$ for complete Tactons, $96.9 \%$ for rhythms, $57.2 \%$ for roughness and $99.8 \%$ for spatial location. The information transmitted was 3.27 bits (9.61 tokens) for complete Tactons, 1.38 bits (2.6 tokens) for rhythm, 0.15 bits (1.1 tokens) for roughness and 1.57 bits (2.96 tokens) for spatial location. These results are shown in Figure 10, alongside the results when the number of roughnesses was reduced.

When the number of roughnesses was reduced to two the percentage correct score for complete Tactons increased from $48.8 \%$ to $80.56 \%$ (Figure 10 ). A one-way ANOVA showed a significant difference in performance $(F=31.47, p<0.01)$, therefore Hypothesis 1 can be accepted.

The information transmitted by complete Tactons increased from 3.27 bits (9.61 tokens) to 3.37 bits (10.3 tokens) when the number of roughnesses was reduced to two. This result indicates that perfect performance could have been achieved with around 10 Tactons. It also shows that decreasing the number of Tactons from 27 to 18 actually increases the amount of information transmitted (from 3.27 bits (9.61 tokens) to 3.37 bits (10.3 tokens)) even though the amount of information input has been decreased (from 4.75 bits (27 tokens) to 4.17 bits (18 tokens)). This increase in information transmission indicates that Hypothesis 3 can be accepted..



Figure 10: Percentage correct scores for complete Tactons and each individual parameter for the 6 participants in the 2 roughness and 3-roughness experiments (with standard deviations).

Appointment types (represented by rhythm) were correctly recognised on average $96.9 \%$ of the time in the three-roughness experiment and $99.1 \%$ of the time in the two-roughness experiment (Figure 10). A one-way ANOVA showed no significant different in performance $(F(1,34)=3.01, p=0.09)$. This result suggests that performance on rhythm identification is comparable in both experiments, and indicates that Hypothesis 4 can be accepted for rhythm. In the three-roughness experiment, the information transmitted by the rhythm parameter was 1.38 bits (2.6 tokens), while in the two-roughness experiment this increased slightly to 1.52 bits, corresponding to 2.86 tokens. This indicates that perfect performance could have been achieved if only 2 rhythms had been used, but that this result was close to perfect.

The results for recognition of importance of appointment (represented by roughness) showed an average recognition rate of $59.47 \%$ in the three-roughness experiment and $82.4 \%$ in the tworoughness experiment (Figure 10). A one way ANOVA showed a significant different in performance $(F(1,22)=6.27, p=0.02)$ therefore Hypothesis 2 can be accepted.Information transmission was calculated for the roughness parameter at 0.15 bits in the three-roughness experiment (1.1 tokens) and increased to 0.33 bits (1.26 tokens) when the number of roughnesses was reduced to two. These results indicate that it may not be possible for users to reliably identify two or more levels of roughness and, therefore, roughness may not be a suitable parameter for Tactons if perfect performance is required, however it may be suitable if recognition of around $80 \%$ is acceptable.

The results for recognition of time until appointment (represented by location) showed an average recognition rate of $99.8 \%$ in the three-roughness experiment and $98.8 \%$ in the two-roughness experiment (Figure 10). A one-way ANOVA showed no significant difference between the two conditions $(F(1,34)=1.90$, $\mathrm{p}=0.18$ ), suggesting that the performance on location identification is comparable in both conditions. This indicates that Hypothesis 4 can be accepted for location. Information transmission was calculated for this parameter at 1.57 bits (2.96 tokens) in the 3-roughness experiment and 1.5 bits (2.8 tokens) in the two-roughness experiment. This indicates that perfect performance could have been achieved if only 2 locations had been used but that this result was very close to perfect.

\subsection{Discussion}

The results of this follow-on study indicate that decreasing the number of roughnesses from three to two significantly improves performance for absolute identification of Tactons encoding three dimensions of information. Although less information is encoded in the Tactons, more information is actually transmitted and, therefore, it is more beneficial to use this smaller set of Tactons. The performance on roughness increased significantly from $57.2 \%$ to $82.4 \%$, indicating that two levels of roughness is the maximum that should be used in Tactons when combined with spatial location and rhythm. However, the performance for roughness is still much lower than that for the other two parameters, therefore it might be worth considering whether a different parameter such as intensity (or a combination of roughness and intensity to provide redundancy) could achieve better performance.

One limitation of this study is that the same participants were used for both experiments, and it is therefore possible that the increase in performance was due in part to the increased training received. Future work should investigate the learning effects to understand the contribution that this may have made to the improvement in performance.

\section{CONCLUSIONS AND FUTURE WORK}

This paper presented two experiments investigating absolute identification of Tactons encoding three dimensions of information. Previous research had investigated identification of 
Tactons encoding two dimensions of information (using rhythm and roughness) and found identification rates of around 70\% [3]; this research extended the amount of information that could be encoded in Tactons by adding a further dimension (encoded in spatial location).

The results of these experiments show that an identification rate of over $80 \%$ can be achieved when three dimensions of information are encoded in Tactons, on the condition that only two levels of roughness are used. When three levels of roughness are used this recognition rate is just $48 \%$. This $81 \%$ performance level exceeds the $71 \%$ identification rate achieved for Tactons encoding two dimensions of information [3], indicating that encoding three dimensions of information in Tactons can be successful. It should be noted, however, that the two-parameter Tactons used three levels of roughness, so performance might also be improved for two-parameter Tactons if the number of roughnesses was reduced to two.

The amount of information transmitted is higher when only two levels of roughness are used, even though the amount of information input has decreased. These results indicate that using less Tactons not only improves performance, but also communicates more information to users. Therefore it can be concluded that it is more efficient to use the smaller set of Tactons.

The information transmission results indicate that near-perfect performance could be achieved if just 10 Tactons were used rather than the existing 18. Future work should investigate how to create this optimum set in order to achieve perfect performance. Although $100 \%$ recognition is not necessary for most of the applications proposed for Tactons, if it could be achieved it would become possible to use these Tactons in safety critical applications, such as alerting systems for firefighters or pilots.

Multi-dimensional Tactons offer the possibility to communicate complex information in a discrete manner and in noisy environments and could therefore be useful in many mobile and wearable applications. The application presented in this paper is the use of Tactons in alerts in a calendar application within a mobile device. Another possible application would be a contextawareness system for blind people, where information about the types of services (restaurant, bank, supermarket) in the surrounding area (as well as information more related to the concerns of visually impaired people, such as the number of stairs leading up to an entrance) could be provided as the user walks around a town.

In order to make the use of multidimensional Tactons in mobile and wearable devices more feasible, future work will consider how to make the spatial location parameter more practicable in a mobile setting. The current use of three Tactors on the forearm might be cumbersome for some mobile applications, but the number of spatial locations could be reduced to two, and the Tactors could be embedded in a watch in one wrist and a bracelet on the other wrist, thus making the system more discrete. In addition, future work will investigate identification of Tactons in a mobile setting, as identification may be affected by being engaged in another activity such as walking.

In conclusion, this work has shown that it is possible to communicate multi-dimensional information through Tactons, which could be used in mobile devices when non-visual information is required. The results of this research could be used by designers to select suitable Tactons for use when designing mobile displays.

\section{ACKNOWLEDGMENTS}

This work was funded by EPSRC studentship GR/PO1175/01 and EPSRC grant GR/S53244. The authors would like to thank Roger Cholewiak for his help with information transmission calculations, and Bruce Mortimer (Engineering Acoustics Inc.) for information on the C-2 Tactors.

\section{REFERENCES}

[1] Immersion VibeTonz System. http://www.immersion.com/mobility/.

[2] Brewster, S. and Brown, L.M. Tactons: Structured Tactile Messages for Non-Visual Information Display, Proc. AUIC 2004, Australian Computer Society (2004), 15 - 23.

[3] Brown, L.M., Brewster, S.A., and Purchase, H.C. A First Investigation into the Effectiveness of Tactons, Proc. World Haptics 2005, IEEE Press (2005), 167-176.

[4] Brown, L.M., Brewster, S.A., and Purchase, H.C. Tactile Crescendos and Sforzandos: Applying Musical Techniques to Tactile Icon Design, to appear in Ext. Abstracts CHI 2006, ACM Press (2006),

[5] Brown, L.M. and Kaaresoja, T. Feel Who's Talking: Using Tactons for Mobile Phone Alerts, to appear in Ext. Abstracts CHI 2006, ACM Press (2006),

[6] Chang, A. and O'Sullivan, C. Audio-Haptic Feedback in Mobile Phones, Proc. CHI 2005, ACM Press (2005), 1264 1267.

[7] Cholewiak, R.W. and Wollowitz, M., The design of vibrotactile transducers, in Tactile Aids for the Hearing Impaired, I. Summers (ed). Whurr Publishers Ltd: London, 1992, 57-82.

[8] Cholewiak, R.W. and Collins, A.A., "Vibrotactile localization on the arm: Effects of place, space and age", Perception and Psychophysics 65, 7 (2003), 1058-1077.

[9] Cholewiak, R.W., Brill, C.J., and Schwab, A., "Vibrotactile localization on the abdomen: Effects of place and space", Perception and Psychophysics 66, 6 (2004), 970-987.

[10] Craig, J.C. and Sherrick, C.E., Dynamic Tactile Displays, in Tactual Perception: A Sourcebook, W. Schiff and E. Foulke (ed). Cambridge University Press, 1982, 209-233.

[11] Miller, G.A., "The Magical Number Seven, Plus or Minus Two: Some Limits on Our Capacity for Processing Information", The Psychological Review, 63, (1956), 81-97.

[12] Senders, V.L., "Measurement and Statistics" Oxford University Press, New York:1958.

[13] Tan, H.Z. and Pentland, A. Tactual displays for wearable computing, Proc. ISWC 1997, IEEE Press (1997), 84-89.

[14] van Erp, J.B.F. and Van Veen, H.A.H.C. A multipurpose tactile vest for astronauts in the international space station., Proc. Eurohaptics 2003(2003), 405-408. 